\title{
Multi-Variable Simulation Decomposition in Environmental Planning: An Application to Carbon Capture and Storage
}

\author{
M. Kozlova ${ }^{1}$ and J. S. Yeomans ${ }^{2 *}$ \\ ${ }^{1}$ School of Business and Management, Lappeenranta University of Technology, Lappeenranta 53850, Finland \\ ${ }^{2}$ OMIS Area, Schulich School of Business, York University, 4700 Keele Street, Toronto, Ontario M3J 1P3, Canada
}

Received 28 January 2019; revised 20 February 2019; accepted 01 March 2019; published online 31 March 2019

\begin{abstract}
Environmental decision-making commonly involves multifaceted problems that demonstrate considerable uncertainty. Monte Carlo simulation approaches have been employed in a variety of environmental planning venues to address these uncertain aspects. Simulation-based outputs are frequently presented in the form of probability distributions. Recently an approach referred to as simulation decomposition (SD) has been introduced that extends the analysis of Monte Carlo results by enhancing the explanatory power of the cause-effect relationships between the multi-variable combinations of inputs and the simulated outputs. SD constructs sub-distributions of the simulation output by pre-classifying some of the uncertain input variables into states, clustering the various combinations of these different states into scenarios, and then collecting simulated outputs attributable to each multi-variable input scenario. Since the contribution of subdivided scenarios to the overall output is easily portrayed visually, SD can highlight and disclose previously unidentified connections between the multi-variable combinations of inputs on the outputs. An SD approach is generalizable to any Monte Carlo model with negligible additional computational overhead and, hence, can be readily used for environmental analyses that employ simulation models. This study illustrates the efficacy of SD in environmental analysis using a carbon capture and storage project from China.
\end{abstract}

Keywords: simulation decomposition, Monte Carlo simulation, environmental decision making under uncertainty, planning and strategy

\section{Introduction}

Environmental planning can prove to be an extremely challenging and complicated activity (Loughlin et al., 2001; Janssen et al., 2010). In the environmental decision-making realm, final decisions are often constructed based not only upon clearly articulated modelling requirements, but also upon numerous political, socio-economic, and environmental requirements that must remain inherently subjective (Loughlin et al., 2001; Zechman and Ranjithan, 2004). Thus, environmental decision-making typically involves complex problems possessing objectives and design requirements that are very difficult to capture when their supporting decision models are formulated (Hipel and Ben-Haim, 1999; Mowrer, 2000; De Kok and Wind, 2003; Brugnach et al., 2007; Matthies et al., 2007; Fuerst et al., 2010; Hipel and Walker, 2011; Castelletti et al., 2012; Lund, 2012; Walker et al., 2012). Environmental planning becomes even more complicated when the system components contain extensive stochastic uncertainties (Baetz, 1990; Yeomans, 2008; Gunalay et al., 2012; Farr et al., 2016; Han et al., 2017).

Monte Carlo simulation approaches have been applied to

${ }^{*}$ Corresponding author. Tel.: +1 (416) 736-5074; fax: +1 (416) 736-5687. E-mail address: syeomans@ @schulich.yorku.ca (J. S. Yeomans).

ISSN: $2663-6859$ print/2663-6867 online

(C) 2019 ISEIS All rights reserved. doi:10.3808/jeil.201900003 a variety of environmental planning settings in order to circumvent some of these uncertainty issues (see, for example: Openshaw and Whitehead, 1985; Ridlehoover, 2004; Byer and Yeomans, 2007; Byer et al., 2009; Byer et al., 2011; Vithayasrichareon and Macgill, 2012; Kim et al., 2013; Farr et al., 2016; Han et al., 2017). An effective simulation analysis needs to capture not only the ranges of realistic possible out-comes, but also the distributional nature of how the identified risks "behave" between the identified extremes (Byer et al., 2011; Kleijnen, 2018). Monte Carlo methods can be used to as sess the potential impacts on different systems based upon the likelihoods of certain events (Byer and Yeomans, 2007; Byer et al., 2009). Since its resulting outputs can be expressed in a distributional format, simulation yields more complex descriptions than a single, deterministic value (Kleijnen, 2018).

However, any subsequent output analysis provides challenges as to how to effectively convey the probabilistic meaning of the stochastic impacts determined. While the impacts could sometimes be expressed numerically as average values and ranges, at other times they can be more effectively represented graphically as probability distributions (Kleijnen, 2018). Although simulation provides an effective means for comparing stochastic system behaviours, it possesses no formal mechanism to actually calculate good system solutions. Thus, the principal decisions associated with simulation must be left to those who examine the uncertainties represented by these sim- 
ulated distributional outputs (Byer et al., 2009). While Monte Carlo methods have been applied to wide variety of problems (Law and Kelton, 2000), simulation and the way its outputs are analyzed have remained relatively static over time (Kleijnen, 2018).

Recently, Kozlova et al. (2016) introduced an ancillary approach referred to as simulation decomposition (SD) that extends the analysis of simulation results by enhancing the explanatory power of the cause-effect relationships between the input variables and the simulation results in multi-variable investment projects. Typically, these simulation-based investment outputs are displayed in the form of histogram distributions. In SD, Kozlova et al. (2016) classified the possible outcomes of selected uncertain variables into states and then used combinations of these states to decompose the simulated output histogram into a number of sub-distributions. The decomposed sub-distributions could then be matched to state-combinations of the variables containing relevant actionable information. Most significantly, these sub-distributions can be superimposed onto an overall output distribution figure to permit a direct visualization of the specific impacts of the decomposed, multivariable groups of input combinations. This visualization of the decomposition facilitates subsequent managerial decisionmaking with regards to the studied investments. The SD approach reflects features of both sensitivity and scenario analysis that are typically employed to complement investment analyses. The method is also generally usable and is not context dependent.

In this paper, the efficacy of SD is extended into an environmental analysis context and is illustrated using a carbon capture and storage (CCS) case taken from Chen et al. (2016). The study is structured to ascertain how a proposed CCS project in China would be affected by whether or not the market price of carbon was independent of a subsidy. A Monte Carlo simulation approach is employed and an SD analysis is applied to the output distributions so that an appropriate visualization of the combined multi-variable input effects becomes evident.

\section{Simulation Decomposition Approach}

Although Monte Carlo techniques enjoy an extensive history of application to a wide spectrum of different problems, the approach-and the way its results have been analyzed-has remained relatively unchanged (Kleijnen, 2018). Kozlova et al. (2016) proposed SD as an enhancement to the explanatory power of simulation by further exploiting the cause-effect relationships inherent between the input variables and the corresponding output. While this section briefly outlines the SD approach, more extensive details and descriptions can be found in Kozlova et al. (2016).

The fundamental steps used for constructing a simulation decomposition are schematically illustrated in Figure 1 and explained below:

1) Identify the input variables $\tilde{V}_{i}$ and the potential distribution ranges that these variables can assume. These variables correspond to those that would "normally" be simulated in a classical Monte Carlo simulation model.
2) Of the input variables from Step 1, identify the key variables of specific interest to the decision-maker and establish relevant states $\tilde{A}_{i j}$ for them (for example: low-high, optimistic-expected-pessimistic, etc.). The identified states highlight different outcome levels in terms of that specific variable.

3) For each key variable in Step 2, determine suitable numerical boundary values $\tilde{B}_{i j}$ corresponding to each of their identified relevant states $\tilde{A}_{i j}$. These boundaries may be either sharp or fuzzy depending upon circumstances.

4) Determine all possible combinations of the states of the key variables. Each of these combinations corresponds to a multi-variable scenario sub-grouping $\tilde{G}_{k}$ of the to-be-decomposed portion of the total simulation. The total number of these multi-variable combinations is necessarily a function of the number of different states identified in Step 2. For example, for two key variables $\tilde{V}_{1}$ and $\tilde{V}_{2}$ in Figure 1 with three states for $\tilde{V}_{1}$ and two states for $\tilde{V}_{2}$, the total number of groups is six.

5) Run the Monte Carlo, assigning the result of each simulated iteration to the output distribution for the "full" simulation while simultaneously noting the specific decomposed sub-distribution corresponding to the input combination $\tilde{G}_{k}$. The identification of the correct sub-distribution for each iteration is achieved by mapping the randomly generated values of each individual input to their corresponding states $\tilde{A}_{i j}$, then mapping these states into the appropriate multi-variable scenario combination $\tilde{G}_{k}$.

6) Construct appropriate output graphs and tables of the simulated outputs. These outputs should display both the overall distributional summaries of the results plus the decomposed summaries projected onto the global figures.

In effect, SD creates sub-distributions of the complete simulation output by pre-classifying some of the uncertain input variables into states, clustering the various combinations of these different states to form scenarios, and then accumulating simulated outputs from each multi-variable scenario. This enables the creation not only of a single "overall" distribution from the simulated results, but simultaneously also a subdivision of this output into several sub-distributions based upon the state combination scenarios. Since the contribution of subdivided scenarios to the overall output is readily visualized, SD can clearly unveil connections between input circumstances (the multi-variable scenarios) and the underlying consequences to which they lead (the simulated results). The SD visualization can be easily implemented by color-coding the overall distribution in accordance with which group (the multi-variable scenario) a particular portion of the distribution belongs to. In essence, the SD distribution is represented by a stacked histogram, where series represent the scenarios that together add up to the overall 'full' distribution.

In other words, SD enables decomposing the overall distribution of outcomes into a set of scenarios. Hence, this enables decision-makers to more effectively visualize and analyze the simulation results. The SD approach is completely generalizable, can be appended as an ancillary extension to any Monte Carlo model with negligible additional computational overhead, 


\begin{tabular}{|c|l|l|l|}
\hline Variable $\tilde{V}_{i}$ & Range & State $\tilde{A}_{i j}$ & Boundary $\tilde{B}_{i j}$ \\
\hline \multirow{2}{*}{$\tilde{V}_{1}: X$} & \multirow{3}{*}[0,100]{} & $\tilde{A}_{11}:$ Low & $\tilde{B}_{11}:[0,30)$ \\
\cline { 3 - 4 } & & $\tilde{A}_{12}:$ Medium & $\tilde{B}_{12}:[30,60)$ \\
\cline { 3 - 4 } & & $\tilde{A}_{13}:$ High & $\tilde{B}_{13}:[60,100]$ \\
\hline \multirow{2}{*}{$\tilde{V}_{2}: Y$} & \multirow{2}{*}{0,1} & $\tilde{A}_{21}:$ True & $\tilde{B}_{21}:\{1\}$ \\
\cline { 3 - 4 } & & $\tilde{A}_{22}:$ False & $\tilde{B}_{22}:\{0\}$ \\
\hline
\end{tabular}

STEP 6

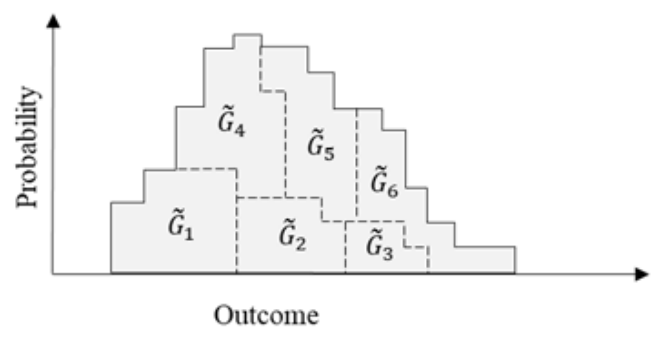

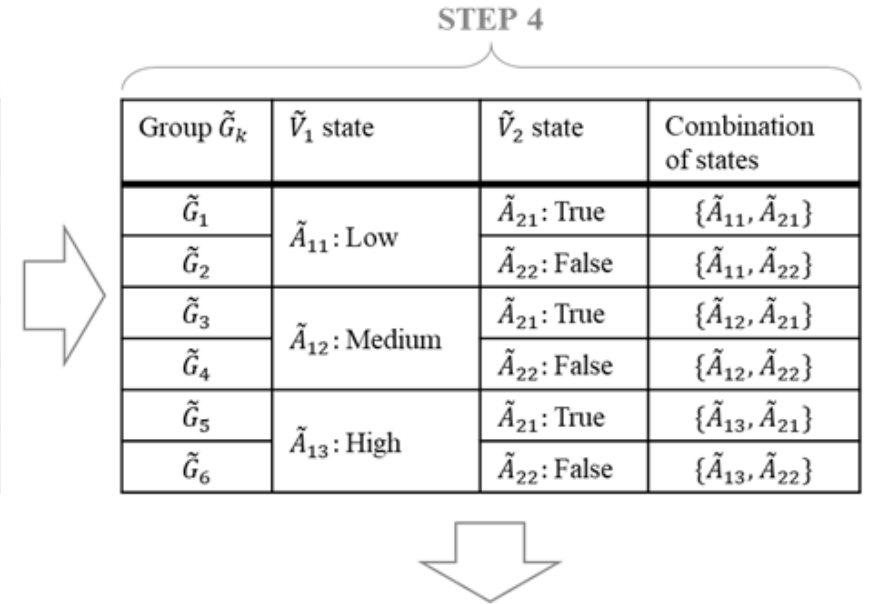

STEP 5

\begin{tabular}{|l|l|l|l|c|}
\hline $\begin{array}{l}\text { Simulation } \\
\text { iteration }\end{array}$ & Output & $\tilde{V}_{1}$ state & $\tilde{V}_{2}$ state & $\begin{array}{l}\text { Resulting } \\
\text { group } \tilde{G}_{k}\end{array}$ \\
\hline 1 & 45 & $15 \in \tilde{A}_{11}$ & $0 \in \tilde{A}_{21}$ & $\tilde{G}_{1}$ \\
\hline 2 & 100 & $79 \in \tilde{A}_{13}$ & $1 \in \tilde{A}_{22}$ & $\tilde{G}_{6}$ \\
\hline 3 & 90 & $75 \in \tilde{A}_{13}$ & $1 \in \tilde{A}_{22}$ & $\tilde{G}_{6}$ \\
\hline 4 & 20 & $7 \in \tilde{A}_{11}$ & $0 \in \tilde{A}_{21}$ & $\tilde{G}_{1}$ \\
\hline$\ldots \ldots$ & \multicolumn{5}{|l}{} \\
\hline 10000 & 75 & $32 \in \tilde{A}_{12}$ & $0 \in \tilde{A}_{21}$ & $\tilde{G}_{3}$ \\
\hline
\end{tabular}

Figure 1. Schematic representation of the simulation decomposition approach.

and can be employed completely independently of the simulation context.

\section{Case Study of Simulation Decomposition Used in Carbon Capture Planning}

In this section, a numerical illustration of a proposed carbon capture and storage (CCS) project in China will be used to show how the SD approach can be effectively extended into environmental analysis. All of the data and parameter estimates have been taken directly from Chen et al. (2016). The broader context of the case and its motivation are presented first, followed by the technical details of the investment modeling and its corresponding simulation model, and then the results of the SD are analyzed. Finally, the benefits of the SD approach are discussed relative to the context of the case.

\subsection{The Case Background}

China is the world's largest emitter of $\mathrm{CO}_{2}$ and generated 9,839 million tonnes (Mt) in 2017 (Global Carbon Project, 2018). This $\mathrm{CO}_{2}$ output constitutes more than one third of all global emissions (Global Carbon Project, 2018). Since coalfired power plants produced $73 \%$ of China's emissions, this corresponds to essentially one quarter of the entire global carbon output (Global Carbon Project, 2018). Therefore, irrespective of any innovation and acceleration in the develop- ment of renewable energy sources, the coal dominated structure of the Chinese power sector will continue to heavily influence global $\mathrm{CO}_{2}$ emissions into the foreseeable future. Consequently, carbon capture and storage of these emissions has been proffered as the relevant decarbonization option (Chen et al., 2016). Unfortunately, previous CCS-only investments have repeatedly proved to be too expensive and unprofitable (see, for example, Rammerstorfer and Eisl, 2011).

After completing several regional pilot projects, China is now on the verge of implementing a national emission trading scheme (Carbon Brief, 2018). Chen et al. (2016), Wang and Du (2016), Fan et al. (2018), and Wang and Qie (2018) have provided CCS policy insights based upon the introduction of this new carbon trading market. All of these insights dictate the need for some form of supplemental subsidy for CCS in addition to the carbon market, itself.

As noted above, coal power plants are responsible for more than $70 \%$ of national $\mathrm{CO}_{2}$ emissions. With carbon capture efficiency currently at approximately $90 \%$, this would effecttively imply a subsidization of $66 \%$ of the overall carbon market price (Chen et al., 2016). Therefore, it is entirely plausible that any extra CCS subsidy of coal power plants would significantly distort the carbon price of the entire market.

To assess the subsidy distortion concept, a Monte Carlo simulation of the CCS investment model will be employed for 
the cases (i) without and (ii) with correlation between the market price and the subsidy. In these investments, the profitability is measured by the net present values (NPV) of the investments (in \$millions). In order to clearly visualize any effect of correlation on the output distributions of the NPVs, SD analysis will be applied in conjunction with the Monte Carlo. In all previous studies, the market price of carbon has been modelled as an independent stochastic variable using geometric Brownian motion that is also independent of the requisite extra subsidy.

\subsection{The Case Modeling}

The illustrative model centres around the construction of a modern $600 \mathrm{MW}$ coal power plant with CCS (Chen et al., 2016). The expense components of the project include the initial infrastructure investment cost, plus the difference in the operations and maintenance costs of the plant with- and without-CCS. In addition, carbon transportation and storage costs are also incorporated. The profit components include revenues from the carbon market and from the subsidy payments. Table 1 provides the values for the parameter estimates employed in the project (Chen et al., 2016). The time horizon for the investment period is set at 35 years.

Table 1. CCS Data Taken from Chen et al. (2016)

\begin{tabular}{lll}
\hline Parameter & Value & Units \\
\hline Installed capacity & 600 & $\mathrm{MW}$ \\
Utilization hours & 5,500 & hours/year \\
Capacity factor & $63 \%$ & Per cent \\
Production & 3,300 & $\mathrm{GWh} / \mathrm{year}$ \\
CO2 emission (rate) & 0.9 & $\mathrm{~kg} / \mathrm{kWh}$ \\
CO2 emission (annual) & $2,970,000$ & tonnes/year \\
CO2 capture efficiency & $90 \%$ & Per cent \\
CO2 capture (annual) & $2,673,000$ & tonnes/year \\
Investment cost (rate) & 538 & \$/kW \\
Investment cost (annual) & 322.8 & \$Millions \\
Operations \& Maintenance cost & $10 \%$ & Per cent \\
(rate) & & \\
Operations \& Maintenance & 56 & \$Millions/year \\
(annual) & & \\
Transport \& storage (rate) & 13 & \$/tonne \\
Transport \& storage (annual) & 35 & \$Millions/year \\
\hline
\end{tabular}

In essence, after installing CCS in year 1, the coal power plant continues to operate over the next 35 years. During this period, costs are incurred as the emissions are captured, transported, and stored. For investment valuation purposes, only cash flows related to the introduction of CCS are considered. This implies that revenues from electricity sales and coal costs are not included in the model, and that basic maintenance costs, except those directly attributable to the introduction of CCS, are omitted.

The key uncertain input variables for the Monte Carlo model are identified as the carbon price and the subsidy payment. The carbon price is generated from a uniform distribution in the range $[0,50] \$ /$ tonne. For decomposition purposes, the three possible relevant states identified for the carbon price together with their corresponding value ranges are: (i) low in the range $[0,10) \$ /$ tonne; (ii) realistic in the range $[10,20) \$ /$ tonne; and (iii) fantastic in the range $[20,50] \$ /$ tonne. Similarly, the subsidy payment is generated from a uniform distribution in the range $[0,0.05] \$ / \mathrm{kWh}$. For the subsidy payment, the two relevant states together with their corresponding value ranges are identified as: (i) low in the range $[0,0.025) \$ / \mathrm{kWh}$; and (iii) high in the range $[0.25,0.05] \$ / \mathrm{kWh}$. Based upon the states identified for these two key variables, there are six possible input combinations, or scenarios, to be employed for decomposing the total simulation. Table 2 provides a complete listing of these scenarios.

Table 2. Six Simulation Decomposition Scenarios for Different Combinations of $\mathrm{CO}_{2}$ Price Levels and Subsidy Amounts

\begin{tabular}{lllll}
\hline Scenario & Subsidy & $\$ / \mathrm{kWh}$ & $\mathrm{CO}_{2}$ Price & $\$ /$ tonne \\
\hline 1 & Low & {$[0,0.025)$} & Low & {$[0,10)$} \\
2 & Low & {$[0,0.025)$} & Realistic & {$[10,20)$} \\
3 & Low & {$[0,0.025)$} & Fantastic & {$[20,50]$} \\
4 & High & {$[0.025,0.05]$} & Low & {$[0,10)$} \\
5 & High & {$[0.025,0.05]$} & Realistic & {$[10,20)$} \\
6 & High & {$[0.025,0.05]$} & Fantastic & {$[20,50]$} \\
\hline
\end{tabular}

The Monte Carlo model with SD was then run twice: (i) for the case where there is no dependency between the carbon price and the subsidy, and; (ii) for the case where a correlation does exist between the price and the subsidy. In each case, the simulation was performed for 10,000 iterations. For the case in which there is a correlation, a revenue outflow is added to represent revenue loss from the carbon market to account for circumstances in which the $\mathrm{CO}_{2}$ price reacts to the subsidy introduction (Chen et al., 2016). This revenue loss is set equal to the subsidy amount weighted by the corresponding share of the emissions coming from the coal power plants in China (namely $73 \%$ as of 2018). For simplicity, the reaction of $\mathrm{CO}_{2}$ prices is assumed to be both immediate and constant over time. For each iteration of the Monte Carlo, the result is assigned to the output distribution for the "full" simulation and the specific decomposed scenario corresponding to the input combination is noted.

\subsection{Results}

The resulting NPV distribution for the case without the price-subsidy dependency is shown in Figure 2, while the distribution for the NPV output with a price-subsidy dependency appears in Figure 3. The entire figures characterize the overall output distributions, while the various shadings correspond to the multi-variable scenarios projected onto the output. The summary statistics for each scenario and both cases with and without the dependency are presented in Table 3.

As can be observed from the figures, the minimum NPVs are equal for both cases. This corresponds to the situation with zero subsidy and zero carbon price. Clearly, the CCS investment is unprofitable without any support. However, the maximum NPVs for the two cases differ quite substantially. The far lower NPVs for the case with correlation are caused by the lower demand in emission allowances due to the direct subsidiza- 
tion from CCS. This leads to a reduced $\mathrm{CO}_{2}$ price which causes the substantially lower $\mathrm{CO}_{2}$ revenues. From an investment perspective, the total magnitude of the impact of this effect can be as much as $\$ 2$ billion for the $600 \mathrm{MW}$ coal power plant (the difference between maximum values of this sixth scenario).

Observing the sub-distribution impacts using the SD scenarios, the different correlation effect cases clearly cause significantly different CCS profitability profiles. For the uncorrelated case, only the low-subsidy-low-carbon-price scenario (Scenario 1 in Figure 2) is always entirely negative. All of the other scenarios (Scenarios $2 \sim 6$ ) show positive NPVs over some, if not most, of their distributions. In the correlated case, only two scenarios, low-subsidy-fantastic-carbon-price and high-subsidy-fantastic-carbon-price (Scenarios 3 and 6 in Figure 3), possess partially positive NPVs-specifically, only under the circumstances in which there is a fantastic market price of carbon does the investment in CCS become profitable. All of the other scenarios (Scenarios 1, 2, 4, 5) remain completely unprofitable throughout their entire distribution ranges. Furthermore, it can be observed from Figure 3 that the correlation effect causes a negative shift of all of the high subsidy scenarios onto the low subsidy scenarios relative to Figure 2. The same can be concluded from Table 3, e.g., the minimum values of low subsidy scenarios (Scenarios $1 \sim 3$ ) are very close between no correlation and correlation cases, while the difference is much higher for the high subsidy scenarios (Scenarios $4 \sim 6$ ). This observation clearly highlights the futility of subsidy increases for situations where there is correlation with the carbon price because the increased subsidy is immediately offset by a corresponding decrease in $\mathrm{CO}_{2}$ price.

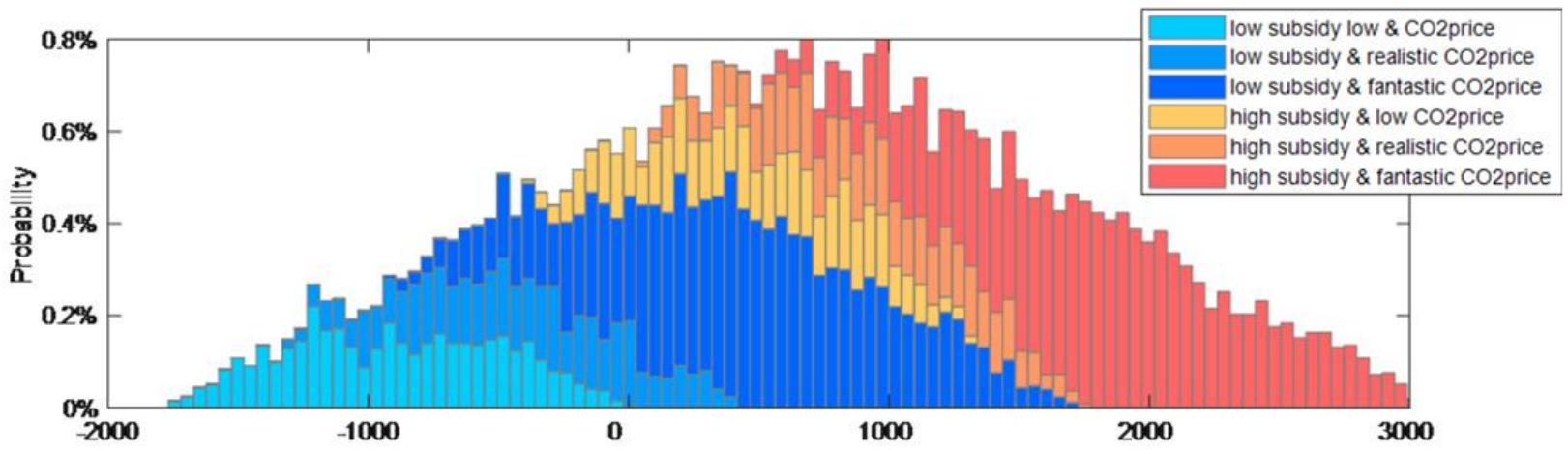

Figure 2. NPV (\$ Millions) of CCS investment for a 600MW coal plant with carbon prices independent of subsidy.

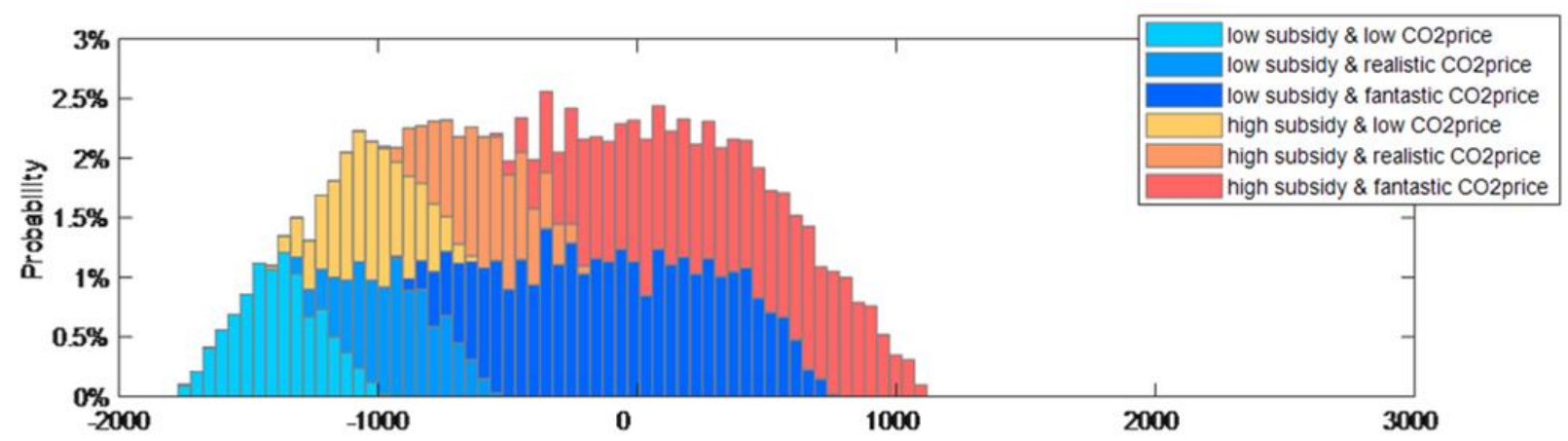

Figure 3. NPV (\$ Millions) of CCS investment for a 600MW coal plant with a linear dependence between carbon prices and the Subsidy.

Table 3. Summary Statistics of Sub-Distributions for the Two Simulations: with and without Correlation (all Values in \$ Millions)

\begin{tabular}{|c|c|c|c|c|c|c|c|c|c|c|}
\hline \multirow{2}{*}{ Scenario } & \multirow{2}{*}{ Subsidy } & \multirow{2}{*}{$\mathrm{CO}_{2}$ Price } & \multicolumn{3}{|c|}{ Without correlation (Figure 2) } & \multicolumn{5}{|c|}{ With correlation (Figure 3 ) } \\
\hline & & & $\min$ & mean & $\max$ & st.dev & $\min$ & mean & $\max$ & st.dev \\
\hline 1 & Low & Low & -1793 & -923 & -34 & 405 & -1796 & -1412 & -1020 & 161 \\
\hline 2 & Low & Realistic & -1343 & -461 & 387 & 410 & -1358 & -964 & -581 & 169 \\
\hline 3 & Low & Fantastic & -922 & 379 & 1689 & 541 & -933 & -104 & 717 & 391 \\
\hline 4 & High & Low & -395 & 438 & 1300 & 406 & -1428 & -1046 & -656 & 160 \\
\hline 5 & High & Realistic & 23 & 860 & 1735 & 401 & -985 & -614 & -221 & 162 \\
\hline 6 & High & Fantastic & 429 & 1744 & 3063 & 545 & -562 & 263 & 1094 & 392 \\
\hline
\end{tabular}


By employing the SD approach, the decomposition highlights that if carbon prices are influenced by a CCS subsidy, then CCS investments are unprofitable except under the situation of an extremely favourable market price. The implication of this outcome is that the extra subsidy would result in significant sunk investments that, if built, would never operate because the carbon price would be too low to justify the loss in power production due to carbon capture. Consequently, the extra subsidy would essentially waste publicly-funded investments with no corresponding emission reduction. Moreover, the results also imply that a CCS subsidy for coal-fired power production directly undermines the carbon market and its usefulness for the other $27 \%$ of emission sources (i.e., the non-coal power), thereby stymying further emission reduction. These findings would seem to correspond congruently to the analogous observations that occur with global carbon trading, in which massive renewable energy support schemes have effectively driven the traded carbon price to zero.

\section{Conclusions}

Environmental planning is often a very complex endeavour that can be heavily influenced by numerous uncertain issues and unquantified factors. These uncertain and inexact dimensions force decision-makers to incorporate many sources of stochastic uncertainty into their decision processes. Any ancillary methods used to support the decision formulation process must include all of these stochastic components while being flexible enough to encapsulate the impacts from the different uncertain elements. One approach frequently used in environmental planning to incorporate stochastic uncertainties is Monte Carlo simulation. In this paper, a Monte Carlo simulation procedure was presented that showed how simulation decomposition could be used to efficiently partition sources of uncertainty into distinct components that permitted the simultaneous influence of multi-variable combinations of several input variables onto the output distributions to be effectively visualized.

The efficacy of an SD approach was illustrated using a carbon capture and storage case from China. The study demonstrated how much the profitability of CCS investments would be affected when the carbon price depends upon an additional subsidy. An investment model and Monte Carlo simulation were employed to illustrate the cases of (i) with and (ii) without a subsidy dependence. To visualize the overall impact from combinations of input variables, the final distribution of the NPVs was decomposed into different scenarios of subsidy and price levels, so that the subsidy dependence effect could become readily observable.

In its stochastic evaluation capacity, SD was shown to be able to efficiently partition the Monte Carlo simulation output distribution into components attributable to specific scenarios of multi-variable input combinations. Unlike other methods, SD permits the simultaneous visualization and projection of these combined multi-variable stochastic uncertainties directly onto the generation of the overall output distribution. The SD method is generally usable and can be utilized independent of the simulation context. Since SD techniques can be adapted into simulation models of a wide variety of problem types, the practicality of this multi-variable partitioning approach can clearly be extended into many different types of environmental, operational, and strategic planning applications containing significant sources of uncertainty. The efficacy of extending SD into a variety of environmental planning situations will be explored in future research.

Acknowledgments. This research was supported in part by grant OGP0155871 from the Natural Sciences and Engineering Research Council and by funding from the Finnish Strategic Research Council, grant number 313396/MFG40 - Manufacturing 4.0.

\section{References}

Baetz, B.W. (1990). Optimization/Simulation sodeling for waste management capacity planning. ASCE Journal of Urban Planning and Development, 116(2), 59-79. https://doi.org/10.1061/(AS CE)0733-9488(1990)116:2(59)

Brugnach, M., Tagg, A., Keil, F., and De Lange, W.J. (2007). Uncertainty matters: Computer models at the science-policy interface. Water Resources Management, 21, 1075-1090. https://doi.org/10. 1007/s11269-006-9099-y

Byer, P.H. and Yeomans, J.S. (2007). Methods for addressing climate change uncertainties in project environmental impact Assessments. Impact Assessment and Project Appraisal, 25(2), 85-99. https:// doi.org/10.3152/146155107X205841

Byer, P.H., Lalani, M.J., and Yeomans, J.S. (2009). Addressing and communicating climate change and its uncertainties in project environmental impact assessments. Journal of Environmental Assessment Policy and Management. 11(1), 29-50. https://doi. org/10.1142/S1464333209003245

Byer, P.H., Colombo, A.F., Sabelli, A., and Ches, C. (2011). Decision Making Under Uncertainties for Adapting to Climate Change in Project Environmental Assessments, Research report to the Canadian Environmental Assessment Agency (CEAA). February 2011.

Carbon Brief. (2018). How Will China's New Carbon Trading Scheme Work? Available at: https://www.carbonbrief.org/qa-how-willchinas-new-carbon-trading-scheme-work (Accessed: January 24, 2019).

Castelletti, A., Galelli, S., Restelli, M., and Soncini-Sessa, R. (2012). Data-driven dynamic emulation modelling for the optimal management of environmental systems. Environmental Modelling \& Software, 34(3), 30-43. https://doi.org/10.1016/j.envsoft.2011. 09.003

Chen, H., Wang, C., and Ye, M. (2016). An Uncertainty Analysis of Subsidy for Carbon Capture and Storage (CCS) Retrofitting Investment in China's Coal Power Plants Using a Real - Options Approach. Journal of Cleaner Production, 137, 200- 212. https: //doi.org/10.1016/j.jclepro.2016.07.074

De Kok, J.L. and Wind, H.G. (2003). Design and application of decision support systems for integrated water management; lessons to be learnt. Physics and Chemistry of the Earth, 28(14-15), 571578. https://doi.org/10.1016/S1474-7065(03)0 0103-7

Fan, J., Xu, M., Wei, S., Zhong, P., Zhang, X., and Yang, Y. (2018). Evaluating the effect of a subsidy policy on Carbon Capture and Storage (CCS) investment decision-making in China - A Perspective Based on the 45Q Tax Credit. Energy Procedia, 154, 22-28. https://doi.org/10.1016/j.egypro.2018.11. 005

Farr, J.V., Faber, I.J., Ganguly, A., Martin, W.A., and Larson, S.L. (2016). Simulation-based costing for early phase life cycle cost analysis: Example application to an environmental remediation project. Engineering Economist, 61(3), 207-222. https://doi.org/ 


\subsection{0/0013791X.2015.1062582}

Fuerst, C., Volk, M., and Makeschin, F. (2010). Squaring the circle? Combining models, indicators, experts and end-users in integrated land-use management support tools. Environmental Management, 46(6), 829-833. https://doi.org/10.1007/s00267- 010-9574-3

Global Carbon Project. (2018). Global Carbon Atlas. Available at: http://www.globalcarbonatlas.org/en (Accessed: January 22, 2019).

Gunalay, Y., Yeomans, J.S., and Huang, G. (2012). Modelling to generate alternative policies in highly uncertain environments: An application to municipal solid waste management planning. Journal of Environmental Informatics, 19(2), 58-69. https://doi.org/10. 3808/jei.201200209

Han, Z., Porras-Alvarado, J.D., and Sun, J. (2017). Monte Carlo simulation-based assessment of risks associated with public- private partnership investments in toll highway infrastructure. Journal of the Transportation Research Board, 2670(1), 59-67. https://doi.org /10.3141/2670-08

Hipel, K., and Ben-Haim, Y. (1999). Decision making in an uncertain world: Information-gap modeling in water resources management. IEEE Transactions on Systems, Man and Cybernetics - part C: Applications and Reviews, 29(4), 506 -517. https://doi.org/10. 1109 15326.798765

Hipel, K.W. and Walker, S.G.B. (2011). Conflict Analysis in Environmental Management. Environmetrics. 22(3), 279-293. https:// doi.org/10.1002/env.1048

Janssen, J.A.E.B., Krol, M.S., Schielen, R.M.J. and Hoekstra A.Y. (2010). The Effect of Modelling Quantified Expert Knowledge and Uncertainty Information on Model Based Decision Making. Environmental Science and Policy, 13(3), 229-238. https://doi.org/10. 1016/j.envsci.2010.03.003

Kim, T.H., Choi, J-S, Park, Y.J., and Son, K. (2013). Life cycle costing: Maintenance and repair costs of hospital facilities using Monte Carlo simulation. The Korean Institute of Building Construction, 13(6), 541-548. https://doi.org/10.5345/JKIBC.2013. 13.6.541

Kleijnen, J.P.C. (2018). Design and analysis of simulation experiments. In: Pilz J., Rasch D., Melas V., Moder K. (eds) Statistics and Simulation. IWS 2015. Springer Proceedings in Mathematics \& Statistics, Cham, Switzerland. 231. pp. 3-22. https://doi.org/10. 1007/978-3-319-76035-3_1

Kozlova, M., Collan, M., and Luukka, P. (2016). Simulation decomposition: New approach for better simulation analysis of multivariable investment projects. Fuzzy Economic Review, 21, 3-18. https://doi.org/10.25102/fer.2016.02.01

Law, A.M. and Kelton W.D. (2000). Simulation Modeling and Analysis (3rd edn), McGraw-Hill, New York, NY.

Loughlin, D.H., Ranjithan, S.R., Brill E.D. and Baugh, J.W. (2001). Genetic algorithm approaches for addressing unmodeled objectives in optimization problems, Engineering Optimization, 33(5), 549569. https://doi.org/10.1080/03052150108940933

Lund J. (2012). Provoking more productive discussion of wicked problems. Journal of Water Resources Planning and Management, 138(3), 193-195. https://doi.org/10.1061/(ASCE)WR.1943-5452. 0000190

Matthies, M., Giupponi, C., and Ostendorf, B. (2007). Environmental Decision Support Systems: Current Issues, Methods and Tools. Environmental Modelling and Software. 22(2), 123-127. https://doi. org/10.1016/j.envsoft.2005.09.005

Mowrer, H. T. (2000). Uncertainty in natural resource decision support systems: Sources, interpretation and importance. Computers and Electronics in Agriculture, 27(1-3), 139-154. https://doi.org/10. 1016/S0168-1699(00)00113-7

Openshaw, B.W. and Whitehead, P. (1985). A Monte Carlo simulation approach to solving multicriteria optimization problems related to plan making, evaluation, and monitoring in local planning. Environment and Planning B: Planning and Design, 12, 321-334. https:// doi.org/10.1068/b120321

Rammerstorfer, M. and Eisl, R. (2011). Carbon capture and storageinvestment strategies for the future? Energy Policy, 39, 7103-7111. https://doi.org/10.1016/j.enpol.2011.08.022

Ridlehoover, J. (2004). Applying Monte Carlo simulation and risk analysis to the facility location problem. The Engineering Economist, 49(3), 237-252. https://doi.org/10.1080/00137910490498942

Vithayasrichareon, P., Macgill, I.F. (2012). A Monte Carlo based decision-support tool for assessing generation portfolios in future carbon constrained electricity industries. Energy Policy, 41, 374392. https://doi.org/10.1016/j.enpol.2011.10.060

Walker, S.G.B., Hipel K.W., and Inohara, T. (2012). Attitudes and Preferences: Approaches to Representing Decision Maker Desires. Applied Mathematics and Computation, 218(12), 6637-6647. https://doi.org/10.1016/j.amc.2011.11.102

Wang, X. and Du, L. (2016). Study of Carbon Capture and Storage (CCS) investment decision-making based on real options for China's coal-fired power plants. Journal of Cleaner Production, 112, 4123-4131. https://doi.org/10.1016/j.jclepro. 2015.07.112

Wang, X. and Qie, S. (2018). When to invest in carbon capture and storage: A perspective of supply chain. Computers \& Industrial Engineering, 123, 26-32. https://doi.org/10.1016/j.cie.2018.06.006

Yeomans, J.S. (2008). Applications of simulation-optimization methods in environmental policy planning under uncertainty. Journal of Environmental Informatics. 12(2), 174-186. https://doi.org/10. 3808/jei.200800135

Zechman, E.M., and Ranjithan, S.R. (2004). An evolutionary algorithm to generate alternatives (EAGA) for engineering optimization problems. Engineering Optimization. 36(5), 539-553. https:// doi.org/10.1080/03052150410001704863 